\title{
Cloud-Computing-Based Rick Control System for Making Information Technology Outsourcing Secure
}

\author{
Ming Shao ${ }^{1, *}$ and Tao Gong ${ }^{2,3}$ \\ ${ }^{1}$ Shanghai University of Engineering Science, Shanghai 200051, China \\ ${ }^{2}$ College of Information S. \& T., Donghua University, Shanghai 201620, China \\ ${ }^{3}$ Engineering Research Center of Digitized Textile \& Fashion Technology, Ministry \\ of Education, Donghua University, Shanghai 201620, China \\ *E-mail:sym@dhu.edu.cn
}

\begin{abstract}
Though the information technology outsourcing (ITO) can help many companies to reduce the total costs of their information technology (IT) projects and seek a more efficient allocation of resources, the ITO-based projects probably have the insecure risks of unexpected costs and even the failure of the whole project. After the quantitative analysis with the principal-agent theory towards the security problem of the ITO-based projects, a new incentive reward-punishment model was proposed in this paper, in order to reduce the ITO insecure risk and generate optimal contract under the asymmetric information condition. The experimental results show that the construction of the new model has positive significance for guiding the outsourcing enterprise management in security.
\end{abstract}

Key Words: Information Technology; Outsourcing; Security; Risk Management

\section{Introduction}

Information technology outsourcing (ITO) is one of the basic outsourcing forms in companies, and it is developed on some computer theories and application technologies. Since the 1990s, the outsourcing has been very popular for the IT projects in many companies, and the typical successful example is Eastman Kodak's externalization of the information systems. Nowadays, the ITO is often regarded as an attractive business proposition to improve the productivity of the IT projects, reduce the costs and increase the competitive ability [1]. The firms can obtain numerous benefits from outsourcing some services of the information systems. At the same time, the ITO perhaps causes an increasing number of decision problems, such as the choice of developing the projects by themselves or outsourcing the projects to others, the selection of the service providers, and the identification of the most appropriate approach to manage the outsourcing contracts [2]. Wrong decision often makes the projects insecure and then will cause much or little loss of the companies. For the outsourcing clients, these issues especially entail the risks that lead to serous negative consequences. Cao and Chen found that the ITO-based projects had high risks, and the degrees of the risks are various in different periods of the projects [3]. Bahli and Rivard proposed a number of risk-assessment frameworks and risk-management strategies for the ITO-based projects [4]. However, very little attention has been paid to design the optimal secure contracts and accomplish the mutual benefit goal of the entire project according to the circumstance with asymmetric information.

In this paper, the past relative researches were firstly analyzed to attain a synthesis of the main issues about the ITO risks and the relative risk managements. In Section 2, the risk factors of the ITO-based projects were modeled to satisfy the security requirements. In Section 3 , the example of the rick control system was designed with the cloud computing and security models, in order to make the information technology outsourcing secure for the IT project of the company. 


\section{Modeling Risk Factors of ITO-Based Projects According to Security}

Because uncertainty is one of the internal causes for the risk $R$, this risk can be described as the following function $f$.

$$
R=f(P, C)
$$

Here, $P$ represents the probability of this uncertainty, and $C$ represents the consequence of the uncertainty.

As for the ITO risk, this uncertainty is the major deterrent of the ITO-based project [5]. This uncertainty originated from the implementation deviation in the process of the ITO-based project. If the enterprises neglect the detailed analysis of the risk and do not use the effective preventive measures against the risk, they will perhaps encounter with much loss.

So it is necessary to analyze the risk factors of the ITO-based projects and the control strategies against the risks, in order to satisfy the security requirements of the ITO industry. The workflow of the ITO-based project is completed by the outsourcers and the vendors. The risk factors refer to the three major objects of the outsourcers, the vendors and the transaction processes.

Firstly, the following risk factors from the outsourcers may cause the security problem of the ITO-based projects.

(1) Difficulty to make the decision about the optimal outsourcer of the ITO-based projects;

(2) Difficulty for the outsourcer to learn new IT knowledge;

(3) Difficulty to combine the IT resources outside the company into the business process of the company.

Secondly, the following risk factors from the vendors may also lead to the security problem of the ITO-based projects.

(1) Lack of IT professional techniques for the vendors;

(2) Business speculation;

(3) Insufficient financial resources.

As a matter of routine, the outsourcers pre-pay a small part of money, which are still not enough for the large-scale outsourcing, when they sign the contracts. Afterwards, if the ITO-based project is interrupted in an emergency, the insecure risks of the outsourcers will be produced. Therefore, the strong economic strengths of the venders should be checked carefully to deal with the possible insecure risks.

Finally, the following risk factors in the transaction process are also insecure for the ITO-based projects.

(1) Much transaction cost out of the limit of the transaction process;

(2) Inappropriate signed contracts of the transaction process.

According to the psychological analysis, the behaviors of the users are affected by the negative affection and the positive reinforcement from the environment. The positive reinforcement contains incentive and rewards, while the negative affection includes inhibition and punishment. In order to avoid the moral hazard of agent under the asymmetric information environment, we use incentive methods to spur the agent to work hard. In addition, the principle agent can set the different levels according to the quality and quantity of the task completed by the agent, and then gives their corresponding incentives. As a result, the principal agent can knit the interests together, and they will also share the risks and the profits. With such a mechanism, the principal agent can prevent the speculative behavior of the agent, and avoid the moral hazard. 
Suppose the effort level of the service supplied by the venders be $t$, and the corresponding revenue generated by the outsourcers be $\pi=A t+\theta$, which means the revenue is actually in proportion to the effort level. The constant $A$ is greater than zero, and this coefficient $A$ is related to the effort level of service supplied by the venders. $\theta$ is the stochastic variable with the expectation 0 and the variance $\delta^{2}$, which satisfies the normal distribution and is generated by exogenous uncertainties. Therefore, $E \pi=A t, \operatorname{var}(\pi)=\delta^{2}$.

The effort cost function of the vender is shown below.

$$
c(t)=\frac{b}{2} \cdot t^{2},
$$

Here, $b$ represents the constant that is greater than zero, and it is the cost coefficient of the vender. If the constant becomes greater, the effort level $t$ will be bigger. The cost function $c(t)$ satisfies the convexity conditions, and $c^{\prime}(x)>0, c^{\prime \prime}(x)>0$.

Suppose $m$ be the benchmark level of hard work, which is decided by the outsourcers in the contract. If the effort level of the vender is less than the variable $m$, then the vender will face the punishments. If the effort level of the vender is greater than the variable $m$, then this person can get the rewards. The corresponding relationship can be represented below.

$$
\psi=\gamma(M-m)
$$

Here, $\gamma$ represents the incentive coefficient $(\gamma>0), M$ represents the monitoring variable, whose value is observed by the outsourcers through supervising the effort level of the venders. Moreover, $M=t+\varepsilon$, because $M$ is the monitoring variable, and this variable inevitably has the observation error $\varepsilon$. The error $\varepsilon$ satisfies the normal distribution, and its expectation is 0 . The variance has the value $\sigma^{2}-\xi$, and the part $\sigma^{2}$ represents the initial variance of the monitoring variable on the condition that the monitoring costs of the outsourcer are zero. $0 \leq \xi \leq \sigma^{2}$, which means the outsourcer can improve the accuracy range of the supervision through some technical tools and methods. For example, the outsourcer can install the intelligent video surveillance system, and hire some professionals to oversee the effort level of the vender.

The supervision cost function of the outsourcer is described below.

$$
d(\xi)=\frac{1}{2} \alpha \xi^{2}
$$

Here, $\alpha$ denotes the coefficient of the supervision cost.

The outsourcer can design the contract, which is represented below.

$$
s=\lambda+\beta \pi+\psi
$$

Here $s$ is the total wages received by vender, $\lambda$ is the basic wages, $\beta$ is a sharing coefficient in the profit, and $\psi$ reflects the changes in wages caused by incentive or punishment measures.

Sometimes, the outsourcer is a risk neuter, while the vender is a risk evader. So the utility function of the vender has the characteristics of constantly evading absolute risk, and this function is represented below. 


$$
U=-e^{\rho w}
$$

Here, $\rho$ is a kind of measure of the absolute risk aversion.

Therefore, the risk cost of the vender is calculated below.

$$
\Delta R C=\frac{1}{2} \rho \operatorname{Var}(s)=\frac{1}{2} \rho\left[\beta^{2} \delta^{2}+\gamma^{2}\left(\sigma^{2}-\xi\right)\right]
$$

Suppose the retention wage of the vender is $\omega_{0}$, and $\omega_{0}$ represents the wage for the vender even if the vender refuses the corresponding contract. The vender will accept the contract as the certainty equivalent wage is greater than the threshold $\omega_{0}$.

Based on the above assumptions, the expected return value of the outsourcer is calculated below.

$$
E_{0}=E(\pi-s-d)=(1-\beta) A t-\lambda+\gamma(t-m)-\frac{1}{2} \cdot \alpha \xi^{2}
$$

The wage of the vender is calculated below.

$$
\omega=s-c=\lambda+\beta(A t+\theta)+\gamma(t+\varepsilon-m)-\frac{b}{2} t^{2}
$$

On the condition of the risk evade, the certainty equivalent wage of vender is calculated below.

$$
E(\omega)-\Delta R C=\lambda+\beta A t+\gamma(t-m)-\frac{b}{2} t^{2}-\frac{1}{2} \rho\left[\beta^{2} \delta^{2}+\gamma^{2}\left(\sigma^{2}-\xi\right)\right] \geq \omega_{0}
$$

As for the vender, he should select the appropriate effort level $t$ in order to ensure the certainty equivalent wage is the maximum, as shown in Figure 1.

$$
t=\frac{A \beta+\gamma}{b}
$$

In the real operation, the outsourcer only pays the wage with the price in the market. Therefore, the outsourcer will select an appropriate $\lambda$. Subsequently, the IR condition and IC condition is inserted into objective function and it can obtain below.

$$
\max E_{0}=A \frac{\beta A+\gamma}{b}-\frac{1}{2} \alpha \xi^{2}-\frac{(\beta A+\gamma)^{2}}{2 b}-\frac{1}{2} \rho\left[\beta^{2} \delta^{2}+\gamma^{2}\left(\sigma^{2}-\xi\right)\right]-\omega_{0}
$$

The corresponding optimum conditions in (12) can be evaluated below.

$$
\begin{aligned}
& \xi=\frac{\rho \gamma^{2}}{2 \alpha} \\
& \beta=\frac{A^{2}\left(\sigma^{2}-\xi\right)}{\delta^{2}+\left(\sigma^{2}-\xi\right)\left(A^{2}+\rho \delta^{2} b\right)} \\
& \gamma=\frac{A \delta^{2}}{\delta^{2}+\left(\sigma^{2}-\xi\right)\left(A^{2}+\rho \delta^{2} b\right)} \\
& t=\frac{\beta A+\gamma}{b}=\frac{A\left[A^{2}\left(\sigma^{2}-\xi\right)+\delta^{2}\right]}{b\left[\left(\delta^{2}+\sigma^{2}-\xi\right)\left(A^{2}+\rho \delta^{2} b\right)\right]}
\end{aligned}
$$


At this point, a Pareto optimal in the contract is obtained in this new model. According to the analysis result, the outsourcer will share his profits to vender in accordance with an appropriate rate after they have a pre-agreed arrangement. This sharing coefficient is $\beta$. If the vender accepts this arrangement, he will pay the effort level $t$ to complete his task. Their cooperation achieves an optimal at this time.

\section{Example and Simulation}

In this example, an outsourcing enterprise wants to outsource its background data process business, so it can focus on its core business. Now there are two venders, i.e. $A$ and $B$. Suppose the effort level is $A$, the risk evade degree is $\rho$, the cost coefficient is $b$, the external random variable variance is $\delta^{2}$ (as shown in Table 1), and the supervision cost coefficient is $\alpha$. In addition, the benchmark level of hard work is $m=0.4$ for both the venders $\mathrm{A}$ and $\mathrm{B}$, Retained income $\omega_{0}=0.3$ and supervision accuracy is 0.001 .

Table 1. Parameters for this Example

\begin{tabular}{|l|l|l|l|l|l|l|}
\hline Influence Factors & $A$ & $\rho$ & $\mathrm{b}$ & $\delta^{2}$ & $\alpha$ & $\sigma^{2}$ \\
\hline Vender $A$ & 0.5 & 0.7 & 0.5 & 0.0225 & 0.3 & 0.0121 \\
\hline Vender $B$ & 0.3 & 0.4 & 0.4 & 0.0225 & 0.4 & 0.0121 \\
\hline
\end{tabular}

According to formulas (14), (15), (16), the following values can be calculated.

$$
\begin{array}{llll}
A: \beta_{1}=0.1094 & \gamma_{1}=0.44 \vdots & t_{1}=0.99 t \\
B: \beta_{2}=0.0424 & \gamma_{2}=0.28 i & t_{2}=0.74 \varepsilon
\end{array}
$$

The formulas (17) and (18) are combined into (12), it can draw the following formulas (19) and (20) within the conditions of tight constraints.

$$
\begin{aligned}
& A: \lambda_{1}=0.0127 \\
& B: \quad \lambda_{2}=0.1100
\end{aligned}
$$

The formulas (19) and (20) are combined into the maximum expected return $E_{0}$.

$$
\begin{aligned}
& A: \max E_{01}=0.6957 \\
& B: \max E_{02}=0.2051
\end{aligned}
$$

Through the above calculation results, it can be seen that the maximum expected returns of the outsourcer are very different due to different operation abilities, risk attitudes, effort cost coefficients and supervision cost coefficients between the two venders. Meanwhile, the different profit sharing coefficients and the different reward-punishment coefficients of the outsourcer are also the reasons. With other equal conditions, the vender $A$ is more suitable than the vender $B$ for the outsourcer.

The example is verified with the BP neural network, as shown in Figure 1. The BP neural network is comprised of three layers, i.e., the input layer, the hidden layer and the output layer. 


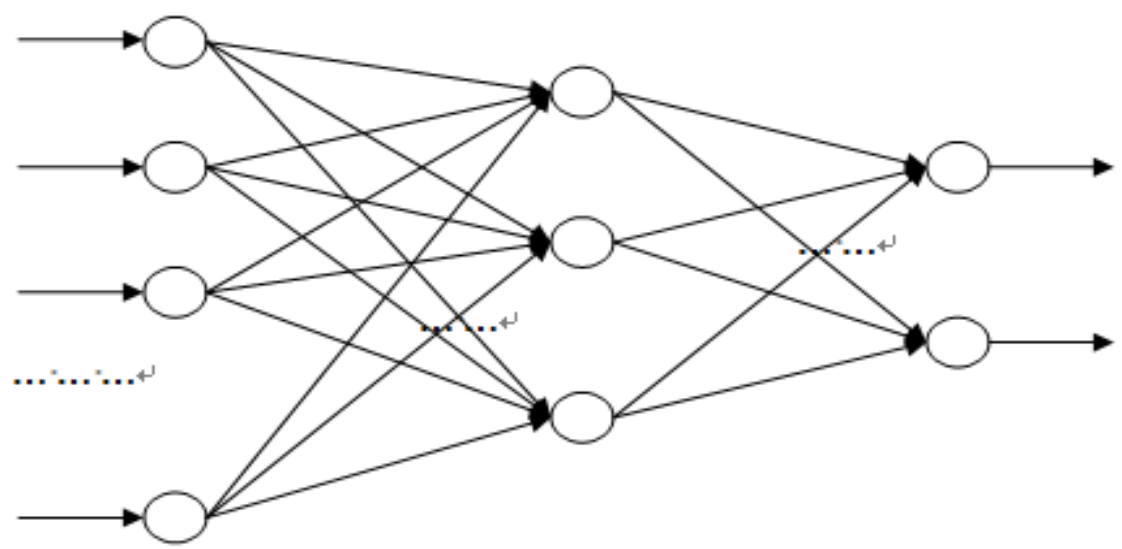

Figure 1. BP Neural Network for Verifying the Example

The computing simulation of this BP neural network has the training results, as shown in Figure 2. Based on the results, we can choose the best company for the ITO task, and then the ITO-based project will get the better fruits.

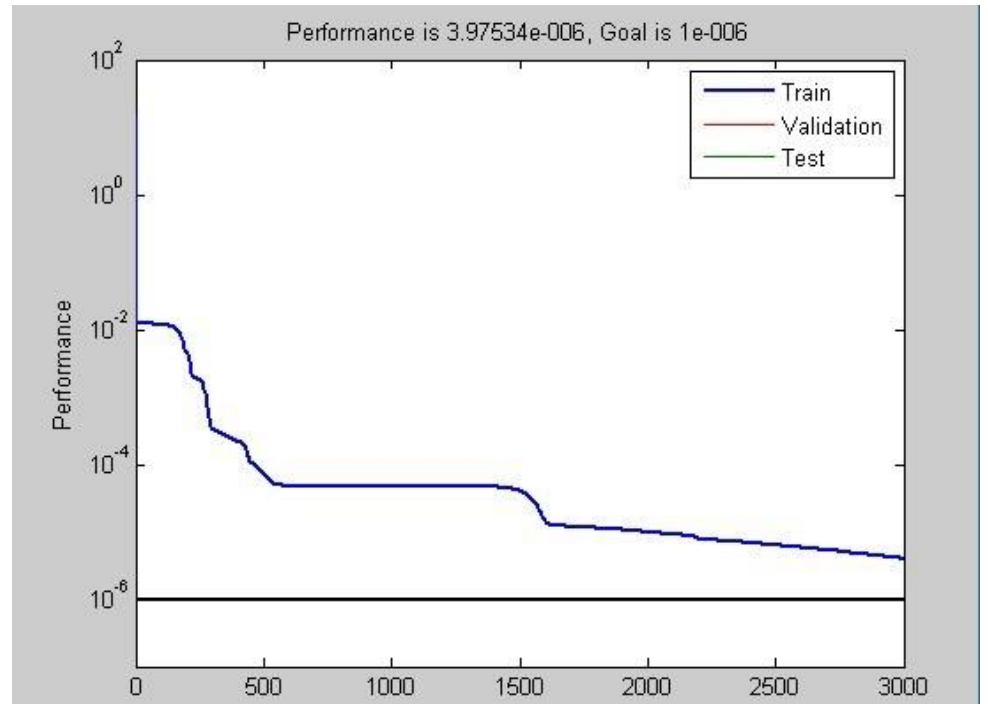

Figure 2. Simulation Results of this BP neural Network for the ITO-based Project

The simulation can be made with the cloud computing tool, such as the CloudSim [6]. This open-source cloud computing simulation software was designed by University of Melbourne (Australia) grid laboratory and Gridbus project. We built a simulation environment (shown in Figure 3) with Oracle 10g JDeveloper for cloud computing in this ITO-based project. 


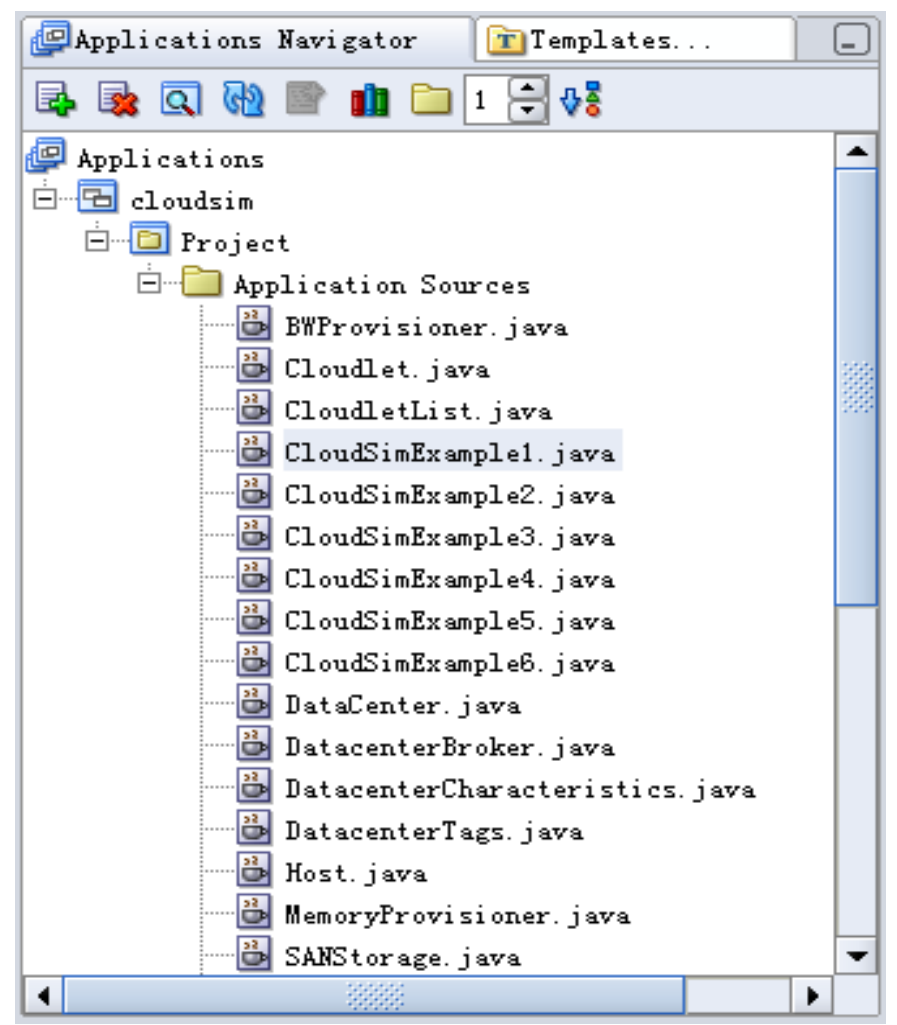

Figure 3. CloudSim-based Simulation of Cloud Computing in the ITO-based Project

\section{Conclusions}

With the rapid development of information technology and global economy, many prominent changes have occurred at the area of international trade in the past. One of the most important changes is the information technology outsourcing (ITO), which is the main security topic of this paper. The ITO insecure risks occur in the implementation steps of the ITO-based projects, and with Principal-Agent theory, a new incentive reward-punishment model was proposed to generate the optimal contract on the asymmetric information condition. The experimental results show that the construction of this new model has positive significance for guiding the secure outsourcing enterprise management.

\section{Acknowledgements}

The author is grateful to the anonymous reviewers for their thorough comments and most useful suggestions. This work was supported in part by the MOE (Ministry of Education in China) Project of Humanities and Social Sciences (Project No.13YJCZH147), the Jiangsu Planned Projects for Postdoctoral Research Funds and the special fund for Shanghai colleges' outstanding young teachers' scientific research projects (Project No.ZZGJD12033).

\section{References}

[1] T. C. Chou, J. R. Chen and S. L. Pan, "The impacts of social capital on information technology outsourcing decisions: a case study of a Taiwanese high-tech firm", International Journal of Information Management, vol. 26, no. 3, (2006), pp. 249-256.

[2] B. A. Aubert, M. Patry and S. Rivard, "A framework for information technology outsourcing risk management", vol. 36, no. 4, (2005), pp. 9-28.

[3] P. Cao and F. J. Chen, "Strategy research on risks avoidance in software outsourcing from provider's perspective", Science and Technology Management Research, vol. 32, no. 19, (2012), pp. 209-213.

[4] B. Bahli and S. Rivard, "Information Technology Outsourcing Risk: A Resource-Based Perspective", Advances in Management Information Systems, vol. 8, no. 11, (2007), pp. 119-134. 
[5] B. A. Aubert, S. Rivard and M. Patry, "A transaction cost model of IT outsourcing", Information \& Management, vol. 41, no. 7, (2004), pp. 921-932.

[6] R. N. Calheiros, R. Ranjan and A. Beloglazov, "CloudSim: A Toolkit for Modeling and Simulation of Cloud Computing Environments and Evaluation of Resource Provisioning Algorithms", Software: Practice and Experience (SPE), vol. 41, no. 1, (2011), pp. 23-50.

\section{Authors}

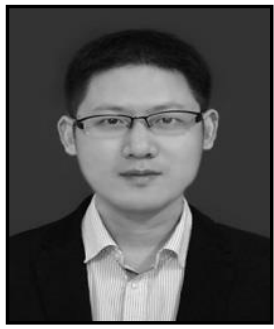

Ming Shao, is a lecturer at Shanghai University of Engineering Science. His research interests include e-commerce, cloud computing, security, information technology outsourcing, etc.

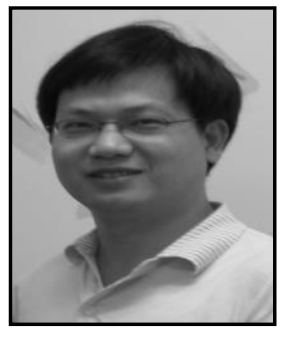

Tao Gong, received the MS degree in Pattern Recognition and Intelligent Systems and $\mathrm{PhD}$ degree in Computer Science from the Central South University respectively in 2003 and 2007.He is an associate professor of computer sciences at Donghua University, China, and he was a visiting scholar at Department of Computer Science and CERIAS, Purdue University, USA. He is the General Editors-in-Chief of the first leading journal Immune Computation in its field, and an editorial board member of some international journals such as Journal of Computers in Mathematics and Science Teaching, International Journal of Security and Its Applications, and International Journal of Multimedia and Ubiquitous Engineering. He is a Technical Committee Chair of ISEEIP 2012, a Publicity Chair of ISA 2012 and a Session Chair of ICAMR 2014. He is also a program committee member of some international conferences such as IEEE ICNC 2011, IEEE BMEI 2011, WMSE 2011, ICARIS 2012, AITS 2012, CCA 2012, ASP 2012, IST 2012, ISA 2012 and SIS2013 etc. $\mathrm{He}$ is a Life Member of Sigma Xi, The Scientific Research Society, a Vice-Chair of IEEE Computer Society Task Force on Artificial Immune Systems, and Chen Guang Scholar of Shanghai. He has published about 100 papers in referred journals and international conferences, and over 20 books such as Artificial Immune System Based on Normal Model and Its Applications etc. He is also a committee member of intelligent robots committee and natural computing committee in the Association of Artificial Intelligence of China. 